\title{
Hypervascular Benign and Malignant Liver Tumors That Require Differentiation from Hepatocellular Carcinoma: Key Points of Imaging Diagnosis
}

\author{
Takamichi Murakami Masakatsu Tsurusaki \\ Department of Radiology, Kinki University Faculty of Medicine, Osaka, Japan
}

\section{Key Words}

Benign liver tumor · Computed tomography $\cdot$ Hepatocellular carcinoma ·

Magnetic resonance imaging

\begin{abstract}
Most liver tumors are benign and hypervascular, and it is important to avoid unnecessary interventions for benign lesions. This review describes the typical and atypical imaging features of common hypervascular benign liver tumors and outlines a general approach to distinguishing between benign and malignant hepatic lesions. There are many types of benign liver tumors that need to be differentiated from hepatocellular carcinoma (HCC). Therefore, it is very important to know the imaging characteristics of benign tumors. Gadolinium ethoxybenzyl diethylenetriamine pentaacetic acid-enhanced magnetic resonance imaging is helpful in diagnosing hypervascular pseudotumors, focal nodular hyperplasia, and nodular lesions associated with alcohol-induced hepatitis. There are also some hypervascular malignant tumors, such as cholangiocarcinoma, cholangiolocellular carcinoma, mixed type tumors, and metastatic liver tumors, which also required differentiation from HCC.
\end{abstract}

Copyright $(2014$ S. Karger AG, Basel

Takamichi Murakami, MD, PhD

Department of Radiology, Kinki University Faculty of Medicine

377-2 Ohno-Higashi, Osaka-Sayama, Osaka 589-8511 (Japan)

Tel. +81 72366 0221, E-Mail murakami@med.kindai.ac.jp 
Table 1. Hypervascular benign liver tumors and hypervascular nontumor lesions

Hypervascular tumors

$\begin{array}{ll}\text { Hepatocyte origin } & \text { HCA } \\ \text { Cholangio cell origin } & \text { Bile duct adenoma } \\ \text { Mesenchymal origin } & \text { Cavernous hemangioma, AML }\end{array}$

Hypervascular nontumor lesions

Hyperplastic Blood flow abnormality Inflammation
FNH

FNH-like nodules

Large regenerative nodules, AP-shunt, Zahn's infarction, peliosis hepatis, confluent fibrosis, abscess, inflammatory pseudotumor, pseudolymphoma

\section{Introduction}

Although liver parenchyma is fed by both the hepatic artery and portal vein, classical hepatocellular carcinoma (HCC) is usually fed by the hepatic artery only. Therefore, imaging of hepatic blood flow, including arterial and portal venous flow, is important for diagnosing liver tumors. Dynamic computed tomography (CT) and magnetic resonance imaging (MRI), CT during arterial portography (CTAP), and CT hepatic arteriography (CTHA) have been developed to evaluate hepatic blood flow [1]. In particular, dynamic CT or MRI is essential for diagnosis of liver tumors. Moreover, MRI with tissue-specific MR contrast media and enhanced ultrasonography with real-time high-spatial-resolution imaging have recently become clinically available [2]. Imaging information enables us to deliver precise diagnoses, but some benign tumors are still difficult to differentiate from malignant lesions. In such cases, patients require invasive examinations, such as biopsy. In this review, we aim to clarify the main imaging features that allow differential diagnosis of benign liver lesions and malignant liver tumors, the latter consisting mainly of HCC.

\section{Key Points of Imaging Diagnosis of Benign Hypervascular Liver Tumors}

There are many types of benign hypervascular tumors that need to be differentiated from hypervascular HCC. When a lesion is diagnosed as benign, it is usually enough to follow it up by imaging examination alone, although some hepatic adenomas requires surgical intervention. The most common benign hypervascular liver tumor requiring differential diagnosis is hemangioma. Other benign hypervascular liver tumors are shown in table 1 and are listed here, starting with the most common: arterioportal (AP) shunt, focal nodular hyperplasia (FNH), hyperplastic nodule associated with portal venous abnormality due to alcohol-induced liver disease or Budd-Chiari syndrome, hepatocellular adenoma (HCA), angiomyolipoma (AML), inflammatory pseudotumor, intrahepatic bile duct adenoma, and rare epithelial and mesenchymal tumors.

\section{Hemangioma}

Hemangioma of the liver is the most common benign liver tumor and is usually of the cavernous hemangioma variety [3]. Hemangioma is considered to be a developmental mal- 


\section{Liver Cancer}

\begin{tabular}{l|l}
\hline \multicolumn{2}{l}{ Liver Cancer 2014;3:85-96 } \\
\hline DOI: 10.1159/000343864 & $\begin{array}{l}\text { C 2014 S. Karger AG, Basel } \\
\text { www.karger.com/lic }\end{array}$ \\
Publisned onIIne: VIay 8,2014 & \multicolumn{1}{l}{ Murakami et al.: Hypervascular Benign and Malignant Liver Tumors That Require }
\end{tabular} Murakami et al.: Hypervascular Benign and Malignant Liver Tumors That Require
Differentiation from Hepatocellular Carcinoma: Key Points of Imaging Diagnosis

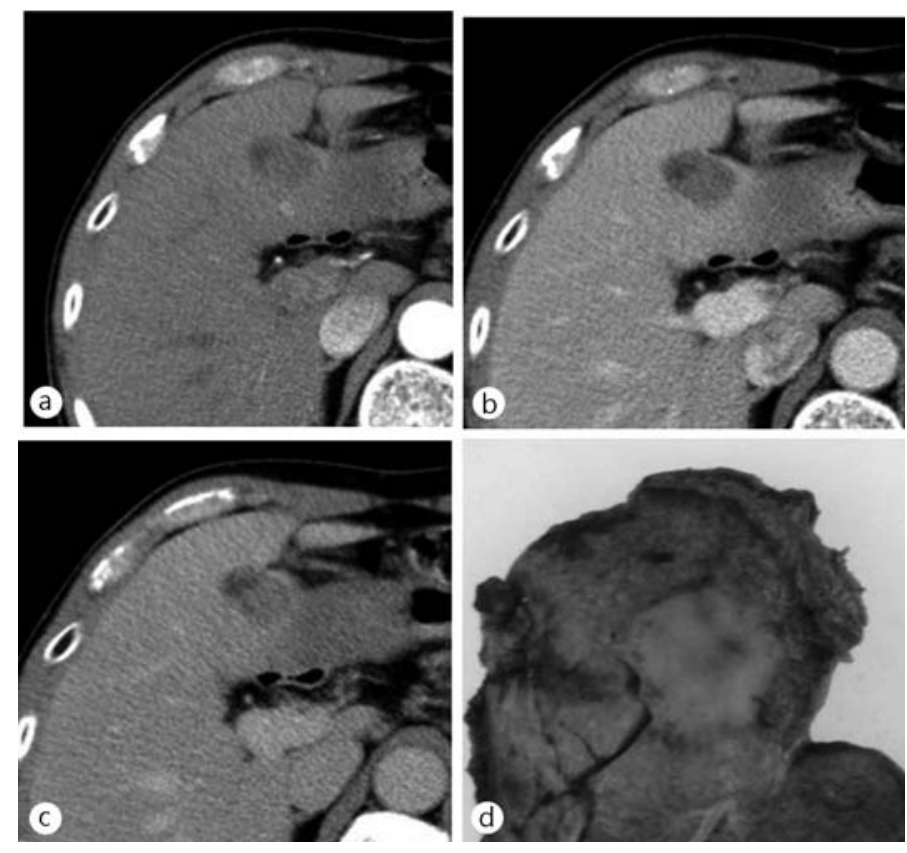

Fig. 1. Sclerosing hemangioma. a Plain CT shows hypoattenuated nodule without fat component in the right lobe. b Arterial phase image of dynamic CT does not show typical peripheral globular enhancement. c Equilibrium phase image of dynamic CT shows prolonged slight enhancement inside the tumor, but not marked enhancement. Therefore, it is difficult to differentiate the lesion from HCC, AML, and metastatic tumor. $\mathbf{d}$ Because chronic hepatitis $\mathrm{C}$ was present as the baseline disease, malignancy could not be excluded. Consequently, hepatic resection was performed. However, the tumor was diagnosed as sclerosing hemangioma with hyalinization inside the tumor.

formation that is usually of no clinical significance. It usually exhibits high echogenicity, but this is not a specific finding for diagnosis. Temporal changes of echogenicity, such as the wax and wane sign, the disappearing sign, and the chameleon sign, are relatively specific findings that are useful for diagnosing hemangioma. Color-Doppler ultrasound imaging has no specific finding for hemangioma and hence has no significant utility in its diagnosis [4].

Dynamic CT and MRI with extracellular contrast media are also very useful. Discontinuous enhancement in the arterial phase (so called spotty, globular, or bright dot enhancement) and prolonged enhancement in the portal venous and equilibrium phases are specific findings for hemangioma [5-8]. The time required for complete contrast fill-in of a hemangioma usually depends on its size. Small lesions may be completely opacified in less than 1 min and undergo homogeneously high attenuation on arterial or portal venous phase images, whereas large lesions may require 20 min or more for complete opacification. Small rapidly enhancing hemangiomas may be associated with adjacent hepatic parenchymal enhancement (staining) related to AP shunts [9]. Most hemangiomas can be diagnosed by dynamic CT; however, it is difficult to diagnose atypical hemangioma with hyalinization and fibrosis inside the tumor (fig. 1). Background diseases, such as a history of HCC or chronic liver disease, may make the diagnosis difficult $[10,11]$.

As stated above, MRI is also a useful modality for diagnosing hemangioma and shows marked high intensity on T2-weighted images. On dynamic MRI with gadolinium diethylenetriamine pentaacetic acid (Gd-DTPA), specific enhancement patterns (spotty, globular, or bright dot enhancement, as stated above) are seen which typically show early hyperin- 


\section{Liver Cancer}

Liver Cancer 2014;3:85-96

DOI: $10.1159 / 000343864$

Publisned online: Vray 8,2014

Murakami et al.: Hypervascular Benign and Malignant Liver Tumors That Require Differentiation from Hepatocellular Carcinoma: Key Points of Imaging Diagnosis
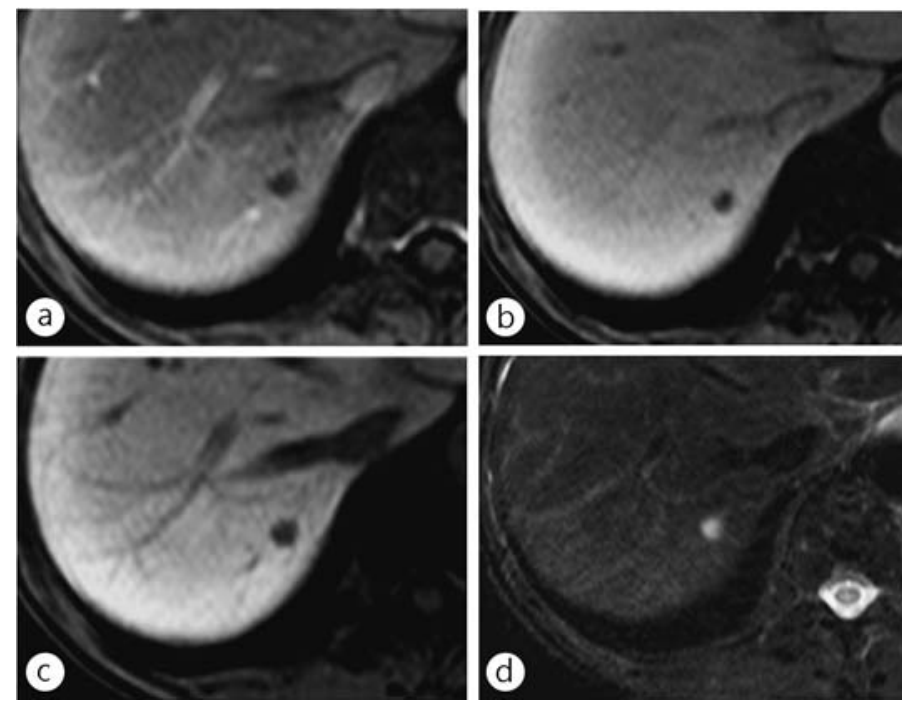

Fig. 2. Cavernous hemangioma on Gd-EOB-DTPA-enhanced MRI. Arterial phase (a), late phase (b), hepatocyte phase (c). Each phase image shows a hypo-enhanced lesion in segment 7 of the liver. The lesion does not show peripheral globular enhancement in the arterial phase or prolonged enhancement in the late phase, which are typical enhancement patterns of hemangioma. However, because the lesion shows very high intensity on the fat-suppressed T2-weighted image (d) despite its relatively small size, hemangioma can be suspected.

tense peripheral nodular enhancement with complete fill-in on delayed images. However, currently, Gd-ethoxybenzyl-DTPA (Gd-EOB-DTPA), a hepatocyte-specific contrast agent, is commonly used on dynamic MRI because Gd-EOB-DTPA-enhanced MRI has been reported to considerably improve diagnosis of HCC and the detection sensitivity of HCC. However, the enhancement pattern of hemangioma on Gd-EOB-DTPA-enhanced MRI is different from that on dynamic CT and MRI with extracellular contrast media, such as iodine and Gd-DTPA. Washout of Gd-EOB-DTPA from the blood pool is much faster than that of iodine contrast media and Gd-DTPA. Thus, hemangioma does not show prolonged enhancement in the portal venous, equilibrium, and hepatocyte phases of Gd-EOB-DTPA-enhanced MRI (fig. 2). On Gd-EOB-DTPA-enhanced MRI, very high intensity on T2-weighted images and specific enhancement patterns in arterial phase images (spotty, globular, or bright dot enhancement) are also important imaging findings for the diagnosis of hemangioma.

\section{Hypervascular Pseudolesions}

Hypervascular pseudolesions are hypervascular enhanced regions in the liver parenchyma on arterial phase images caused by AP-shunts. There is no tumor in the hypervascular region. As is often the case in chronic liver diseases such as hypervascular HCC, the differential diagnosis is important. AP-shunts are often seen in the subcapsular area of the liver and have a wedge shape with no washout of contrast medium in the portal venous phase. The peripheral portal branch is seen in the hypervascular region (dot sign). These specific findings are useful to differentiate hypervascular pseudolesions from hypervascular HCC. However, some AP shunts have a round shape, and this complicates the differential diagnosis. Imaging findings such as the gradual decrease of hyperenhancement and no corona enhancement may be helpful for differential diagnosis $[12,13]$. AP shunts usually exhibit iso-enhancement in hepatocyte-phase images on Gd-EOB-DTPA-enhanced MRI, indicating no decrease of GdEOB-DTPA uptake in the liver parenchyma. However, it should be noted that AP shunts may 


\section{Liver}

show hypo-enhancement in hepatocyte-phase images because liver function can be impaired by long-standing AP-shunts [14].

\section{Focal Nodular Hyperplasia}

FNH is a benign hypervascular tumor arising from the normal liver parenchyma. It occurs primarily in young women, is solitary in $75-80 \%$ of cases, and is often discovered incidentally on abdominal CT or ultrasound examinations $[15,16]$. The typical imaging findings of FNH are a central scar, intratumoral centrifugal arteries from the center, and the presence of Kupffer cells and the proliferation of cholangiole, among others.

On contrast-enhanced CT, FNHs undergo marked enhancement during the arterial phase, becoming appreciably hyperattenuating relative to the hepatic parenchyma. Moreover, one or more large feeding hepatic arteries, small central and septal arteries, and early draining veins often can be identified in large lesions. During the hepatic parenchymal phase, FNH usually becomes isoattenuating or nearly isoattenuating relative to normal hepatic parenchyma $[17$, 18].

A measure of the central scar can be detected by any imaging modality, especially as a high-intensity focusing area on T2-weighted MR images. The central scar, which is identified on MRI in approximately $30-50 \%$ of cases, is characteristically hypointense on T1-weighted images and hyperintense on T2-weighted images. However, FNH without a central scar is not uncommon.

The intratumoral centrifugal arteries from the center seen on angiography, the so called spoke-wheel appearance, and direct venous drainage are specific imaging findings for FNH. Enhanced ultrasound is useful because it can detect the hemodynamics easily. However, the specific imaging findings can be seen only in $40 \%$ of FNH. In the past, the presence of Kupffer cells and cholangioles was evaluated by technetium-99m-labeled sulfur colloid scintigraphy, but recently, hepatic tissue-specific MR contrast media, such as superparamagnetic iron oxide (SPIO) or Gd-EOB-DTPA, have been used for this purpose. Iso- or high intensity or sometimes ring enhancement of the FNH in the hepatocyte image of Gd-EOB-DTPA-enhanced MR images (accumulation of Gd-EOB-DTPA in the tumor) is a very specific finding. This finding is helpful to differentiate FNH from HCC because the extracellular properties of these agents can demonstrate the typical vascular enhancement pattern of FNH on dynamic post-contrast images. In addition, delayed imaging demonstrates uptake of the agent by hepatocytes within the lesion, demonstrating the hepatocellular origin of the mass $[19,20]$. On Gd-EOB-DTPAenhanced MR images, the area composed of hyperplastic hepatic nodules is hyperintense and the central scar is hypointense on hepatocyte-phase images, showing a typical imaging pattern of stellate or lacelike hypointensity within a hyperintense nodule (fig. 3) [21].

Fibrolamellar HCC, which usually arises in relatively young people, is an important malignant disease to differentiate from FNH. The points of differential diagnosis between FNH and fibrolamellar HCC should be noted. Fibrolamellar HCC shows heterogeneous enhancement in all phase images of dynamic CT and MRI. It also has central scar, as FNH does, but its central scar shows hypointensity on T2-weighted images due to its fibrous nature. Fibrolamellar HCC often has calcification in the tumor (68\%) and lymph node metastasis, whereas the scar in FNH is rarely calcified (1.4\% of lesions) [22]. In addition, HCC usually does not show delayed enhancement after Gd-EOB-DTPA administration.

\section{Nodular Lesions Associated with Alcohol-Induced Chronic Hepatic Disease (FNH-Like Nodules) \\ Since Glund et al. [23] reported that hyperplastic nodules developed during the follow- up period in alcoholic men with micronodular cirrhosis, several investigators have reported hyperplastic nodules associated with alcoholic liver cirrhosis [24-29]. It was also pointed out}



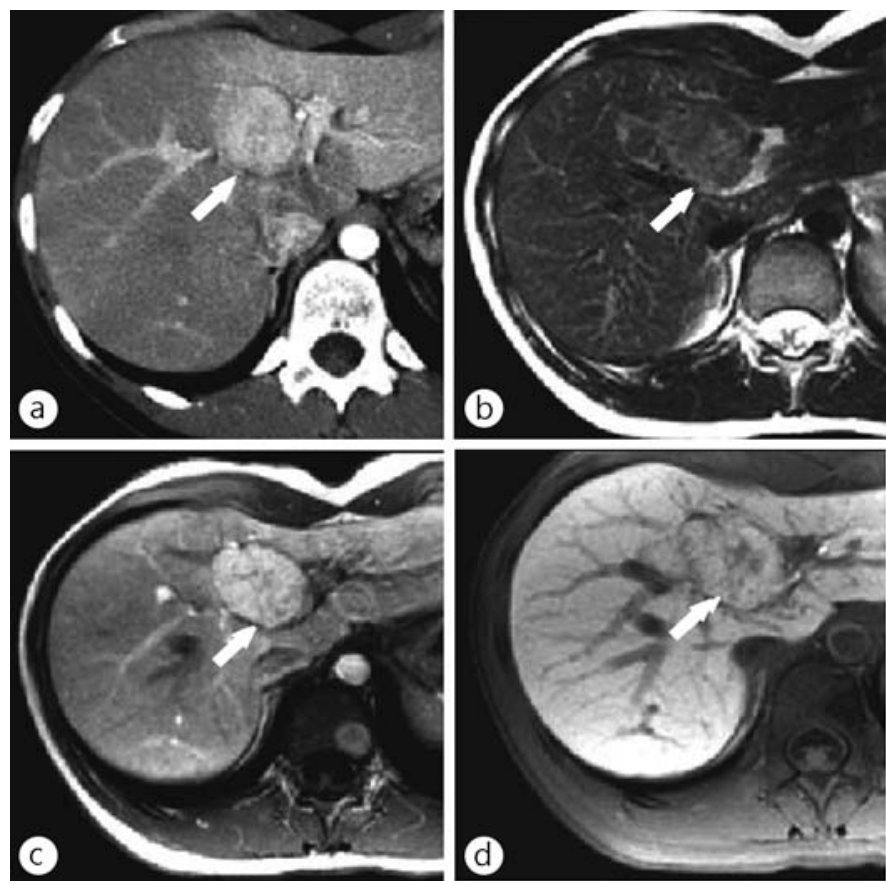

Fig. 3. FNH. a Arterial phase image of dynamic CT shows marked enhanced tumor, but the central scar is not enhanced (arrow). b T2-weighted image shows mild high intensity lesion (arrow) with central marked high intensity region indicating the central scar. c Arterial phase image of Gd-EOB-DTPA-enhanced MRI shows marked enhanced tumor (arrow), but the central scar is not enhanced. d Hepatocyte phase image of Gd-EOB-DTPA-enhanced MRI also shows slightly higher enhancement of the tumor relative to the liver parenchyma (arrow), and the central scar as hypoenhancement. Because the tumor with a central scar takes up Gd-EOB-DTPA, the likely diagnosis is FNH.

that these nodules had some histopathological similarities to FNH in non-cirrhotic liver; consequently, these nodules are called FNH-like nodules or lesions[29]. FNH-like lesions can also be seen in liver showing hemodynamic changes due to disease, such as Budd Chiari syndrome.

FNH-like nodules are small hypervascular lesions, usually less than $2 \mathrm{~cm}$ in diameter. They usually show marked arterial enhancement despite their small size. Because other hypervascular tumors such as metastasis, hepatocellular adenoma, or HCC may mimic FNHlike nodules, it is often difficult to make a confident diagnosis of FNH-like nodules based on vascularity alone, especially in atypical cases. However, FNH-like nodules do not show corona enhancement on CT hepatic angiography. Moreover, they take up SPIO and Gd-EOBDTPA and commonly show iso- or hyperintensity in the hepatocyte phase of Gd-EOB-DTPA-enhanced MRI, in contrast to malignant liver tumors and hepatocellular adenoma, the majority of which demonstrate hypointensity [30]. Just as for FNH, SPIO- and Gd-EOB-DTPAenhanced MRI are usually helpful for the diagnosis of FNH-like nodules (fig. 4). In cases seen in association with chronic liver disease, differential diagnosis from HCC becomes important and is sometimes difficult. In such cases, delicate follow up or biopsy may be required.

\section{Hepatocellular Adenoma}

HCA is a benign tumor that requires differentiation from HCC and FNH. The etiology of HCA is unknown, but it may be induced by hormonal medicines, such as those used in some 


\section{Liver Cancer}

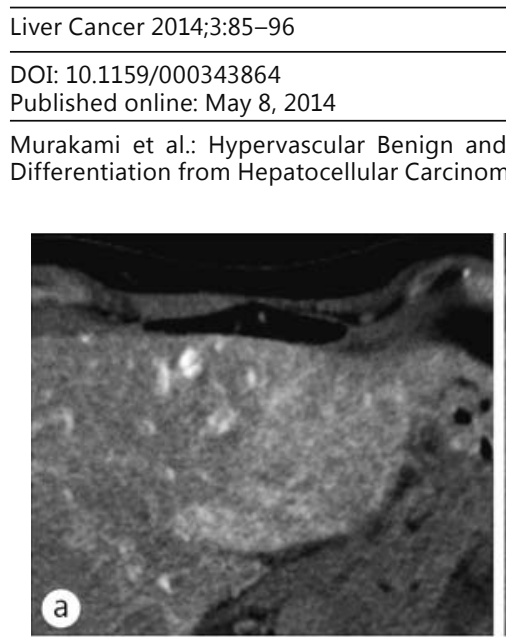

(C) 2014 S. Karger AG, Basel www.karger.com/lic Murakami et al.: Hypervascular Benign and Malignant Liver Tumors That Require
Differentiation from Hepatocellular Carcinoma: Key Points of Imaging Diagnosis
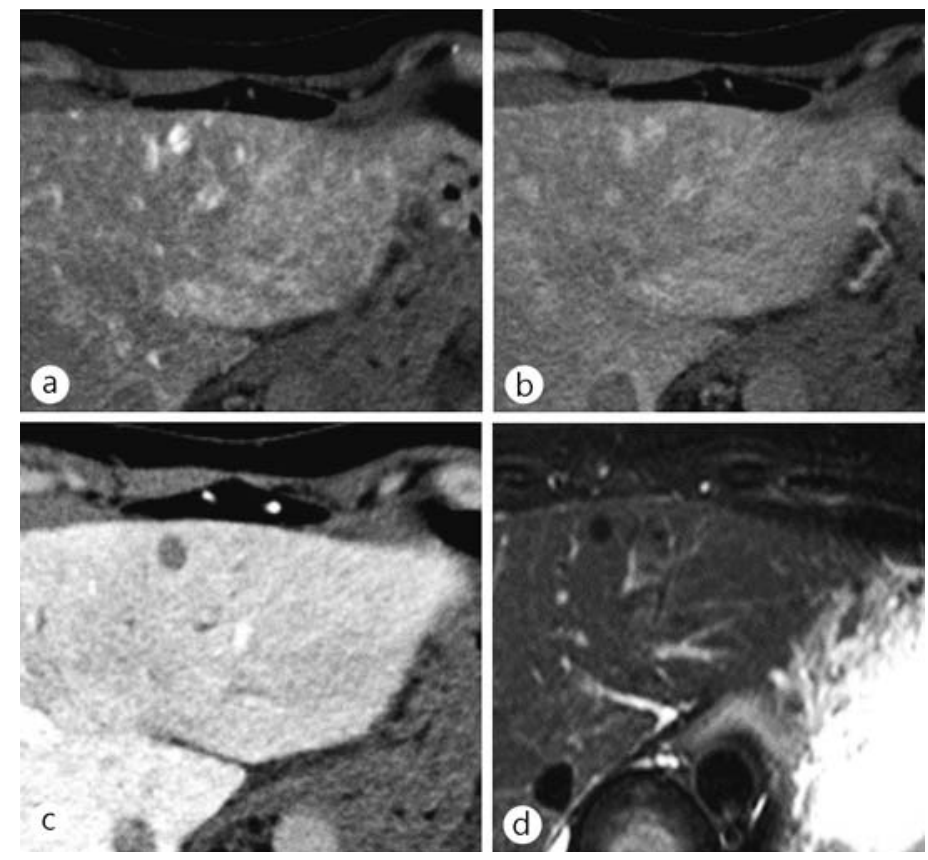

Fig. 4. FNH-like nodule associated with alcohol-induced chronic hepatic disease. a Early phase of CTHA shows small hyperenhanced lesion in the left lobe. b Late phase of CTHA shows washout of contrast medium in the lesion, but still higher enhancement than the liver parenchyma. The lesion does not show corona enhancement. c CTAP shows clear hypoattenuated lesion indicating reduced portal venous perfusion. d SPIO-enhanced MRI shows the tumor as a hypointense lesion, an indication that the tumor contains Kupffer cells and takes up SPIO more readily than the surrounding liver parenchyma.

contraceptives, and can sometimes be seen in livers of patients with some types of glycogen storage disease.

Typical imaging findings of HCA are homogeneous arterial enhancement and prolonged mild enhancement with smooth margins. However, it can sometimes show heterogeneous patterns if intratumoral hemorrhage is present. HCA shows heterogeneous signal intensity on T1- and T2-weighted images, which reflect intratumoral hemorrhage and necrosis. HCA usually exhibits hypointensity on hepatocyte phase images of Gd-EOB-DTPA-enhanced MRI (fig. 5). These findings are useful for differentiating HCA from FNH but do not facilitate differentiation from HCC.

In the 2010 WHO classification, HCA was divided into four subtypes based on molecular biology (hepatocyte nuclear factor $1 \alpha$-inactivated type, $\beta$-catenin-activating mutation type, inflammatory type, and unclassified type) [31]. Inflammatory HCA is the most common subtype and accounts for about $30-50 \%$ of all HCAs. These tumors are mainly seen in women, in association with obesity, hepatic steatosis, diabetes mellitus, glycogenesis (in particular, type I glycogen storage diseases), and alcohol abuse. More than $90 \%$ of women with HCA have a history of hormonal contraceptive use. There are few reports about specific imaging findings of each HCA subtype. In 2008, Laumonier et al. [33] were the first to publish the typical MRI features of HCA according to the subtype classification. A homogeneous dropout of signal on the out-of-phase T1-weighted sequence had a sensitivity of $86.7 \%$ and a specificity of $100 \%$ for hepatocyte nuclear factor $1 \alpha$-inactivated HCA, whereas this dropout was absent or only focal (heterogeneous) in inflammatory HCA. Moreover, Grazioli et al. reported that marked 

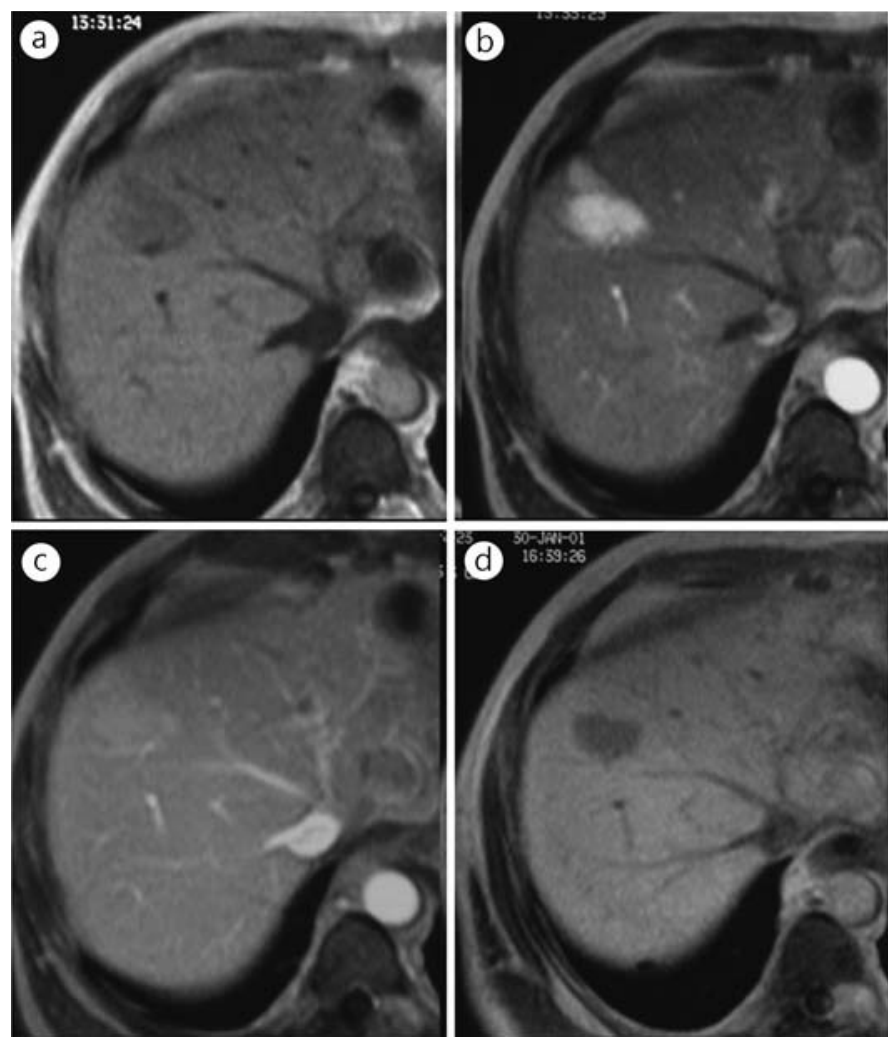

Fig. 5. Hepatocellular adenoma. a Pre-contrast T1-weighted image shows a hypointense lesion in segment 8. b Arterial phase image of Gd-EOB-DTPA-enhanced MRI shows homogenous marked enhancement of the tumor. c Late phase image of Gd-EOB-DTPA-enhanced MRI shows washout of contrast medium in the tumor, but still slightly higher enhancement of the tumor relative to the surrounding liver parenchyma without a central scar. d Hepatocyte phase image of Gd-EOB-DTPAenhanced MRI also shows marked hypointensity of the tumor relative to the liver parenchyma, without central a scar. HCA was strongly suspected; however, malignancy could not be excluded. Consequently, hepatic resection was performed. The tumor was diagnosed as hepatocellular adenoma (inflammatory HCA).

hyperintensity on T2-weighted images associated with delayed persistent enhancement has a sensitivity as high as $85 \%$ and a specificity of $87 \%$ for the diagnosis of inflammatory HCA. Because there are sinusoidal dilatation, inflammatory component and ductal reaction in adenomas, mainly in the periphery, those are seen as areas of hypointensity in the hepatocyte phase image [20]. Several authors have reported that a faint scar may be a possible sign of $\beta$-catenin-mutated HCA [32, 33].

\section{Angiomyolipoma}

Hepatic AML is a rare disease and consists of hypervascular tumor with a fat component. The fat component can be detected by CT and ultrasonography. When the fat deposition is very minor, chemical-shift MR imaging is useful because of its sensitivity. Other benign liver tumors with fat components are myelolipoma, pseudolipoma, and lipoma; these lesions are rare and are usually hypovascular. Malignant liver tumor with a fat component is usually well-differentiated HCC with fatty metamorphosis, which is also usually hypovascular, allowing it to be differentiated from AML. AML without a fat component is hypervascular and sometimes needs to be differentiated from hypervascular HCC. Venous drainage of AML is 


\section{Liver Cancer}

\begin{tabular}{|c|c|}
\hline Liver Cancer 2014;3:85-96 & \\
\hline $\begin{array}{l}\text { DOI: } 10.1159 / 000343864 \\
\text { Publisned onIIne: Ivlay } 8,2014\end{array}$ & $\begin{array}{l}\text { (c) } 2014 \text { S. Karger AG, Basel } \\
\text { www.karger.com/lic }\end{array}$ \\
\hline
\end{tabular}

Murakami et al.: Hypervascular Benign and Malignant Liver Tumors That Require Differentiation from Hepatocellular Carcinoma: Key Points of Imaging Diagnosis
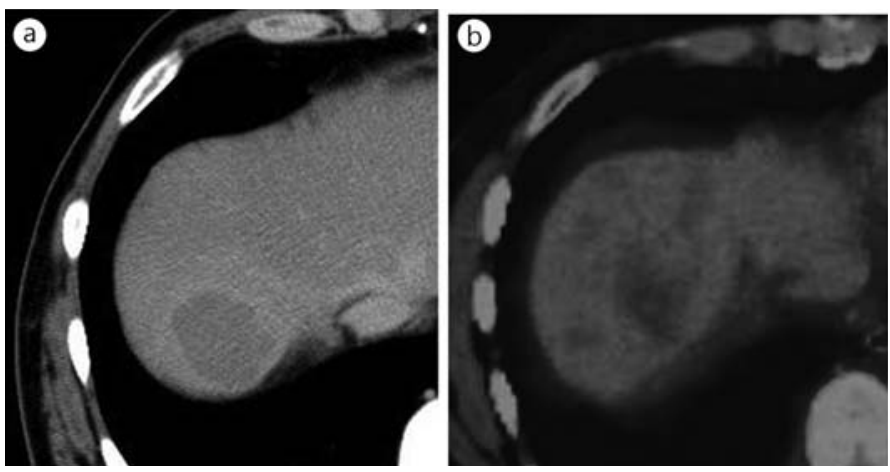

Fig. 6. Pseudolymphoma of the liver. a On contrast-enhanced CT, a hypoattenuating tumor with a slightly hyperattenuating rim surrounding the tumor is shown in the right lobe of the liver. b FDG-PET scan shows high accumulation of FDG in the tumor (SUVmax; maximal standard uptake value is 7.4).

via the hepatic vein, but that of HCC is via the portal vein. These finding can be detected by enhanced ultrasonography or angiography, and sometimes can be detected by dynamic CT or MRI.

\section{Inflammatory Tumor}

Inflammatory tumors include liver abscess, pseudolymphoma and some subtypes of inflammatory pseudotumor. Liver abscess usually shows typical clinical symptoms with specific imaging findings, such as segmental stain, the rim sign, and the double target sign, and thus its diagnosis is not particularly difficult. However, small abscesses $(<1 \mathrm{~cm})$ may show enhancement and mimic hemangioma.

Pseudolymphoma exhibits arterial enhancement and washout of contrast medium in the late phase. It also shows hypointensity of the hepatocyte phase of Gd-EOB-DTPA-enhanced MRI; consequently, differentiating it from HCC may be difficult. Pseudolymphoma usually shows obvious hypoecho and high uptake on fluorodeoxyglucose positron emission tomography (FDG-PET, fig. 6). These findings are helpful points to differentiate pseudolymphoma from HCC.

Inflammatory pseudotumor rarely exhibits clinical symptoms. Park et al. found that contrast-enhanced CT scans indicated poorly defined peripheral enhancement at the arterial phase in $82.5 \%$ of inflammatory pseudotumor cases and indicated poorly defined hyperattenuating lesions with internal hypoattenuating areas at the equilibrium phase in $77.0 \%$ of cases. Gd-DTPA-enhanced MRI revealed poorly defined peripheral rim-like enhancement $77.8 \%$ of cases) [34]. However, the imaging findings may vary according to the inflammatory stage. It may sometimes be difficult to differentiate inflammatory pseudotumor from other focal lesions such as atypical HCC, intrahepatic cholangiocarcinoma, metastatic tumors, or abscess that might show similar enhancement patterns during CT or MRI [35]. Follow up examination enables the correct diagnosis to be made, but sometimes a biopsy is required.

\section{Key Points of Imaging Diagnosis of Malignant Hypervascular Liver Tumors Other Than HCC}

The malignant hypervascular liver tumors that require differentiation from HCC include cholangiocarcinoma, cholangiolocellular carcinoma, mixed tumor containing HCC and cholan- 

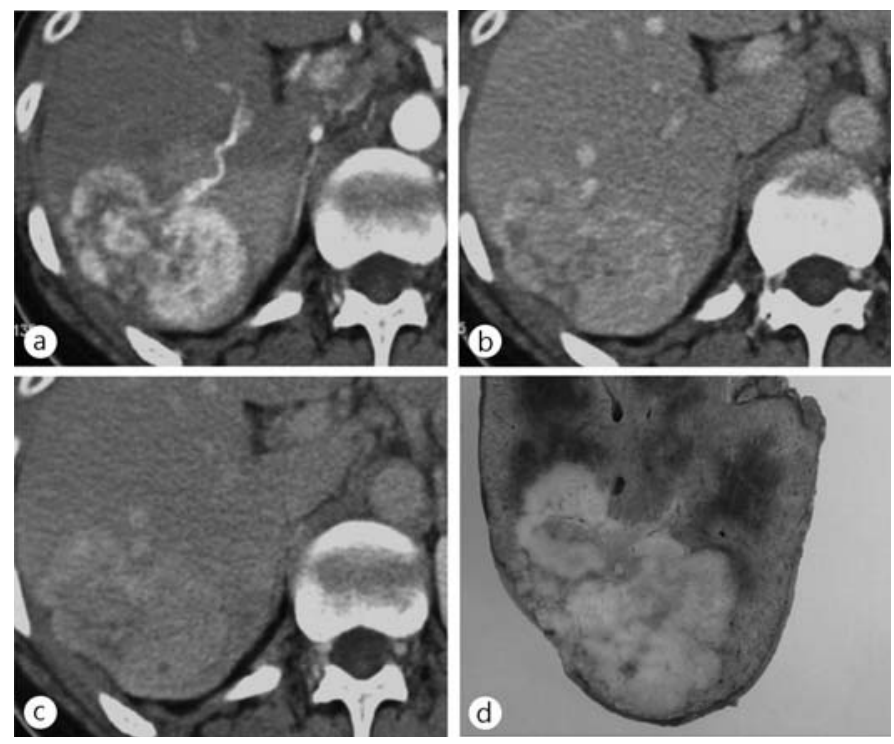

Fig. 7. Cholangiolocellular carcinoma. a Arterial phase image of dynamic CT shows a heterogeneous hypervascular tumor in the right lobe. b Portal venous phase image of dynamic CT shows wash out, but the lesion is still hyperattenuating. There are vascular (portal vein) penetrating signs in the tumor. c Equilibrium phase image of dynamic CT shows prolonged slight enhancement of the tumor. Therefore, it is difficult to differentiate the tumor from HCC; however, its enhancement pattern is not typical of HCC. d The resected specimen shows a lobulated tumor without capsule and with vascular penetration in the tumor. The tumor was thus pathologically diagnosed as cholangiolocellular carcinoma.

giocarcinoma, and metastatic tumors. It is easy to diagnose these lesions when they exhibit specific imaging findings. We indicate some of the key diagnostic points below.

\section{Cholangiocarcinoma}

Overall, $30 \%$ of cholangiocarcinomas arise against a background of chronic hepatic disease, and they are reported to show hypervascularity in a high number of cases [36]. Therefore, it is important to differentiate cholangiocarcinoma from hypervascular HCC, which also usually arises in patients with chronic hepatic disease. Cholangiocarcinoma shows peripheral bile duct dilatation and late enhancement due to intratumoral fibrosis without a capsule. These imaging findings are differential diagnostic points from HCC.

\section{Cholangiolocellular Carcinoma}

Cholangiolocellular carcinoma is classified into "mixed liver cancer with stem cell features, cholangiocellular subtype" in 2010 WHO classification [31]. It shows arterial enhancement in the same way that hypervascular HCC does, but also shows prolonged enhancement, no capsule, and signs of vascular penetration in the tumor [37]. These imaging findings are useful for differentiating cholangiolocellular carcinoma from HCC (fig. 7).

\section{Mixed Type Liver Cancer}

Mixed type liver cancer is classified into the classical subtype, which is composed of HCC and cholangiocarcinoma, and a subtype with stem cell features. Imaging findings vary according to the compositional balance of HCC and cholangiocarcinoma. Consequently, it is often difficult to differentiate mixed type liver cancer from HCC. 


\section{Metastatic Liver Tumor}

Metastatic liver tumor of adenocarcinoma, such as colon cancer or pancreatic cancer, often shows doughnut-like ring enhancement due to its mucinous and fibrous components. As a result, it needs to be differentiated from cholangiocarcinoma. Metastatic liver tumors of renal cell carcinoma, breast cancer, and neuroendocrine tumors, for example, exhibit hypervascularity and have to be differentiated from HCC. When the primary lesion is known, metastatic tumors can be easily diagnosed.

\section{Summary}

We described the key points of imaging diagnosis of benign and malignant hypervascular liver tumors that have to be differentiated from HCC. We may encounter benign lesions that are difficult to differentiate from malignant lesions, but by evaluating background factors, clinical information, and the specific imaging findings stated above, such liver tumors can be diagnosed precisely.

\section{References}

1 Lee JM, Yoon JH, Joo I, Woo HS: Recent advances in CT and MR imaging for evaluation of hepatocellular carcinoma. Liver Cancer 2012;1:22-40.

-2 Joo I, Choi BI: New paradigm for management of hepatocellular carcinoma by imaging. Liver Cancer 2012;1:94-109.

-3 Dietrich CF, Mertens JC, Braden B, Schuessler G, Ott M, Ignee A: Contrast-enhanced ultrasound of histologically proven liver hemangiomas. Hepatology 2007;45:1139-1145.

$\checkmark 4$ Perkins AB, Imam K, Smith WJ, Cronan JJ: Color and power Doppler sonography of liver hemangiomas: a dream unfulfilled? J Clin Ultrasound 2000;28:159-165.

-5 Ashida C, Fishman EK, Zerhouni EA, Herlong FH, Siegelman SS: Computed tomography of hepatic cavernous hemangioma. J Comput Assist Tomogr 1987;11:455-460.

6 Freeny PC, Marks WM: Hepatic hemangioma: dynamic bolus CT. AJR Am J Roentgenol 1986;147:711-719.

7 Itai Y, Furui S, Araki T, Yashiro N, Tasaka A: Computed tomography of cavernous hemangioma of the liver. Radiology 1980;137:149-155.

-8 Leslie DF, Johnson CD, MacCarty RL, Ward EM, Ilstrup DM, Harmsen WS: Single-pass CT of hepatic tumors: value of globular enhancement in distinguishing hemangiomas from hypervascular metastases. AJR Am J Roentgenol 1995;165:1403-1406.

-9 Hanafusa K, Ohashi I, Himeno Y, Suzuki S, Shibuya H: Hepatic hemangioma: findings with two-phase CT. Radiology 1995;196:465-469.

10 Tsurusaki M, Kawasaki R, Yamaguchi M, Sugimoto K, Fukumoto T, Ku Y, Sugimura K: Atypical hemangioma mimicking hepatocellular carcinoma with a special note on radiological and pathological findings. Jpn J Radiol 2009;27:156-160.

$\checkmark 11$ Cheng HC, Tsai SH, Chiang JH, Chang CY: Hyalinized liver hemangioma mimicking malignant tumor at MR imaging. AJR Am J Roentgenol 1995;165:1016-1017.

12 Ueda K, Matsui O, Kawamori Y, Kadoya M, Yoshikawa J, Gabata T, Nonomura A, Takashima T: Differentiation of hypervascular hepatic pseudolesions from hepatocellular carcinoma: value of single-level dynamic CT during hepatic arteriography. J Comput Assist Tomogr 1998;22:703-708.

13 Tsurusaki M, Sugimoto K, Fujii M, Fukuda T, Matsumoto S, Sugimura K: Combination of CT during arterial portography and double-phase CT hepatic arteriography with multi-detector row helical CT for evaluation of hypervascular hepatocellular carcinoma. Clin Radiol 2007;62:1189-1197.

$\checkmark 14$ Motosugi U, Ichikawa T, Sou H, Sano K, Tominaga L, Muhi A, Araki T: Distinguishing hypervascular pseudolesions of the liver from hypervascular hepatocellular carcinomas with gadoxetic acid-enhanced MR imaging. Radiology 2010;256:151-158.

15 Carlson SK, Johnson CD, Bender CE, Welch TJ: CT of focal nodular hyperplasia of the liver. AJR Am J Roentgenol 2000;174:705-712.

16 Nguyen BN, Fléjou JF, Terris B, Belghiti J, Degott C: Focal nodular hyperplasia of the liver: a comprehensive pathologic study of 305 lesions and recognition of new histologic forms. Am J Surg Pathol 1999;23:14411454.

17 Brancatelli G, Federle MP, Katyal S, Kapoor V: Hemodynamic characterization of focal nodular hyperplasia using three-dimensional volume-rendered multidetector CT angiography. AJR Am J Roentgenol 2002;179:81-85. 
18 Hussain SM, Terkivatan T, Zondervan PE, Lanjouw E, de Rave S, Ijzermans JN, de Man RA: Focal nodular hyperplasia: findings at state-of-the-art MR imaging, US, CT, and pathologic analysis. Radiographics 2004;24:3-17, discussion 18-19.

19 Huppertz A, Haraida S, Kraus A, Zech CJ, Scheidler J, Breuer J, Helmberger TK, Reiser MF: Enhancement of focal liver lesions at gadoxetic acid-enhanced MR imaging: correlation with histopathologic findings and spiral CT - initial observations. Radiology 2005;234:468-478.

-20 Grazioli L, Bondioni MP, Haradome H, Motosugi U, Tinti R, Frittoli B, Gambarini S, Donato F, Colagrande S: Hepatocellular adenoma and focal nodular hyperplasia: value of gadoxetic acid-enhanced MR imaging in differential diagnosis. Radiology 2012;262:520-529.

21 Kim MJ, Rhee HJ, Jeong HT: Hyperintense lesions on gadoxetate disodium-enhanced hepatobiliary phase imaging. AJR Am J Roentgenol 2012;199:W575-W586.

-22 Caseiro-Alves F, Zins M, Rahmouni A, Vilgrain V, Menu Y, Mathieu D, Mahfouz A-E: Calcification in focal nodular hyperplasia: a new problem for differentiation from fibrolamellar hepatocellular carcinoma. Radiology 1996;198:889-892.

23 Gluud C, Christoffersen P, Eriksen J, Wantzin P, Knudsen BB: Influence of ethanol on development of hyperplastic nodules in alcoholic men with micronodular cirrhosis. Gastroenterology 1987;93:256-260.

-24 Sugihara S, Nakashima 0, Kiyomatsu K, Ijiri M, Edamitsu O, Kojiro M: A case of liver cirrhosis with a hyperplastic nodular lesion. Acta Pathol Jpn 1990;40:699-703.

25 Terada T, Kitani S, Ueda K, Nakanuma Y, Kitagawa K, Masuda S: Adenomatous hyperplasia of the liver resembling focal nodular hy perplasia in patients with chronic liver disease. Virchows Arch A Pathol Anat Histopathol 1993;422:247-252.

26 Quaglia A, Tibballs J, Grasso A, Prasad N, Nozza P, Davies SE, Burroughs AK, Watkinson A, Dhillon AP: Focal nodular hyperplasia-like areas in cirrhosis. Histopathology 2003;42:14-21.

27 An HJ, Illei P, Diflo T, John D, Morgan G, Teperman L, Theise N: Scirrhous changes in dysplastic nodules do not indicate high-grade status. J Gastroenterol Hepatol 2003;18:660-665.

28 Libbrecht L, Bielen D, Verslype C, Vanbeckevoort D, Pirenne J, Nevens F, Desmet V, Roskams T: Focal lesions in cirrhotic explant livers: pathological evaluation and accuracy of pretransplantation imaging examinations. Liver Transpl 2002;8:749-761.

29 Nakashima O, Kurogi M, Yamaguchi R, Miyaaki H, Fujimoto M, Yano H, Kumabe T, Hayabuchi N, Hisatomi J, Sata M, Kojiro M: Unique hypervascular nodules in alcoholic liver cirrhosis: identical to focal nodular hyperplasia-like nodules? J Hepatol 2004;41:992-998.

30 Yoneda N, Matsui O, Kitao A, Kita R, Kozaka K, Koda W, Kobayashi S, Gabata T, Ikeda H, Sato Y, Nakanuma Y: Hepatocyte transporter expression in FNH and FNH-like nodule: correlation with signal intensity on gadoxetic acid enhanced magnetic resonance images. Jpn J Radiol 2012;30:499-508.

31 Bosman FT, Hruban RH, Theise ND: WHO Classification of Tumours of the Digestive System. Lyon, IARC, 2010.

-32 Laumonier H, Bioulac-Sage P, Laurent C, Zucman-Rossi J, Balabaud C, Trillaud H: Hepatocellular adenomas: magnetic resonance imaging features as a function of molecular pathological classification. Hepatology 2008;48:808-818.

-33 van Aalten SM, Thomeer MG, Terkivatan T, Dwarkasing RS, Verheij J, de Man RA, Ijzermans JN: Hepatocellular adenomas: correlation of MR imaging findings with pathologic subtype classification. Radiology 2011;261:172-181.

34 Park JY, Choi MS, Lim YS, Park JW, Kim SU, Min YW, Gwak GY, Paik YH, Lee JH, Koh KC, Paik SW, Yoo BC: Clinical features, image findings, and prognosis of inflammatory pseudotumor of the liver: a multicenter experience of 45 cases. Gut Liver 2014;8:58-63.

35 Kitajima K, Shiba H, Nojiri T, Uwagawa T, Ishida Y, Ichiba N, Yanaga K: Intrahepatic cholangiocarcinoma mimicking hepatic inflammatory pseudotumor. J Gastrointest Surg 2007;11:398-402.

-36 Kim SA, Lee JM, Lee KB, Kim SH, Yoon SH, Han JK, Choi BI: Intrahepatic mass-forming cholangiocarcinomas: enhancement patterns at multiphasic CT, with special emphasis on arterial enhancement pattern - correlation with clinicopathologic findings. Radiology 2011;260:148-157.

37 Asayama Y, Tajima T, Okamoto D, Nishie A, Ishigami K, Ushijima Y, Kakihara D, Aishima S, Taketomi A, Honda H: Imaging of cholangiolocellular carcinoma of the liver. Eur J Radiol 2010;75:e120-e125. 